\title{
QUALIDADE DE FRUTO DA ACEROLEIRA CV. OLIVIER EM DOIS ESTÁDIOS DE MATURAÇÃ̃O ${ }^{1}$
}

\author{
ELISA ADRIANO ${ }^{2}$, SARITA LEONEL ${ }^{3}$, REGINA MARTA EVANGELISTA ${ }^{4}$
}

RESUMO - A acerola é um fruto de grande potencial econômico e nutricional devido ao seu alto teor de vitamina $\mathrm{C}$, destacando-se como alimento funcional. É comercializada principalmente na forma de polpa congelada e fruto in natura. O objetivo deste estudo foi avaliar a qualidade dos frutos da aceroleira cv. Olivier em dois estádios de maturação. Para tanto, foram colhidas amostras de frutos em um pomar comercial no município de Junqueirópolis-SP. Foram realizadas a determinação da cor externa dos frutos e análises das características químicas de teor de sólidos solúveis, $\mathrm{pH}$, acidez titulável, açúcares redutores, 'ratio' e ácido ascórbico de frutos semi-maduros e maduros. Os resultados obtidos permitiram concluir que os frutos semimaduros apresentaram maior acidez total, menor teor de sólidos solúveis e menor concentração de açúcares; no entanto, estes frutos apresentaram maiores teores de vitamina $\mathrm{C}$, expressa em ácido ascórbico. Portanto, quando se buscam altos índices de vitamina $\mathrm{C}$, os frutos devem ser colhidos num estádio de maturação menos avançado, com coloração alaranjada. O estudo demonstrou também que a cv Olivier produz frutos com características adequadas tanto para o mercado in natura quanto para a indústria, apresentando boa coloração e características químicas dentro dos padrões para esta fruta.

Termos para indexação: Malpighia emarginata DC., cor, ácido ascórbico.

\section{FRUIT QUALITY OF BARBADOS CHERRY TREES CV. OLIVIER IN TWO STAGES OF MATURITY}

\begin{abstract}
Barbados cherry is a fruit of high economic and nutritional potential due to its high vitamin $\mathrm{C}$ content, standing out as a functional food. It is marketed mainly in the form of frozen pulp and fresh fruit. The aim of this study was to evaluate the fruit quality of

barbados cherry trees cv. Olivier in two stages of maturity. Fruits samples were harvested in a commercial orchard in Junqueirópolis, SP, Brazil. The exterior color of the fruit was observed and analysis of the chemical characteristics of pulp, such as soluble solids, $\mathrm{pH}$, acidity, reducing sugars, 'ratio' and ascorbic acid content were performed in semi-ripe and ripe fruits. The results showed that the semi-ripe fruits had higher acidity, lower soluble solids content and lower sugar content, however these fruits had higher levels of vitamin C, expressed as ascorbic acid. Therefore, when seeking high levels of vitamin $C$, the fruit must be harvested in a less advanced stage of maturity, showing orange color. The study also showed that the cv. Olivier produces fruits with characteristics suitable for both fresh market and processing, with good color and chemical characteristics within the standards.
\end{abstract}

Índex terms: Malpighia emarginata DC., color, ascorbic acid.

\section{INTRODUÇÃO}

A aceroleira (Malpighia emarginata DC.) é bastante adaptável aos mais diversos climas e pode ser encontrada em várias regiões do planeta, porém o seu cultivo comercial concentra-se em regiões tropicais e subtropicais (KONRAD, 2002).

No Brasil, a cultura distribui-se nas regiões Nordeste, Norte, Sul e Sudeste (RITZINGER;
RITZINGER, 2004), e a produtividade média dos pomares brasileiros é de 29,65 toneladas de acerola por hectare ao ano, equivalente a $59,3 \mathrm{~kg} / \mathrm{planta} / \mathrm{ano}$ (AGRIANUAL, 2010).

A acerola é comercializada principalmente na forma de polpa congelada e fruto in natura e entra no mercado para os consumidores que preferem sucos naturais. Devido ao seu alto teor de vitamina C, é considerado um produto de alta qualidade, destacando-se

\footnotetext{
${ }^{1}$ Resumo Sinfruit 052 - Simpósio Internacional de Fruticultura - Avanços na Fruticultura (17 a 21 Outubro)

${ }^{2}$ Doutoranda em Agronomia - Horticultura, Departamento de Produção Vegetal, FCA/UNESP, Botucatu- SP, Fazenda Lageado, Botucatu-SP, CEP: 18610-000. E-mail: elisaadrianojd@hotmail.com

${ }^{3}$ Professor Adjunto, Departamento de Produção Vegetal, FCA/UNESP, Botucatu-SP. E-mail: sarinel@fca.unesp.br

${ }^{4}$ Professor Adjunto, Departamento de Produção Vegetal, FCA/UNESP, Botucatu-SP. E-mail: evangelista@fca.unesp.br
} 
no campo dos alimentos funcionais. Outros produtos de acerola que podem ser encontrados no mercado interno são: acerola em pó, acerola com vitamina $\mathrm{E}$, cápsulas medicinais de vitamina $\mathrm{C}$ pura, geleias $\mathrm{e}$ doces (MANICA et al., 2003).

O fruto da aceroleira é uma drupa de tamanho, forma e peso variáveis. A casca é fina e delicada, o tamanho varia de 1 a $2,5 \mathrm{~cm}$ de diâmetro, e o peso, de 3 a $15 \mathrm{~g}$. Quanto à cor, os frutos maduros podem apresentar diferentes tonalidades, que vão do amarelo ao vermelho intenso ou roxo. O sabor varia de levemente ácido a muito ácido (JUNQUEIRA et al., 2004).

Trata-se de um fruto climatérico, ou seja, pode amadurecer na planta ou após a colheita, quando colhido imaturo. É bastante suculento ( $73 \%$ do peso do fruto corresponde à polpa) e com teor de ácido ascórbico variando entre 1.000 a $5.000 \mathrm{mg} / 100 \mathrm{~g}$ de suco, o que corresponde a até 80 vezes a quantidade encontrada em limões e laranjas (TEIXEIRA: AZEVEDO, 1994). Além da vitamina C, é uma fonte razoável de pró-vitamina $\mathrm{A}$, também contém vitaminas do complexo B, como tiamina (B1), riboflavina (B2) e niacina (B3) e minerais como cálcio, ferro e fósforo (RITZINGER: RITZINGER, 2004).

Com relação aos frutos da cultivar Olivier, estes são grandes, têm em média $29,7 \mathrm{~mm}$ de diâmetro e $24,2 \mathrm{~mm}$ de altura, com peso médio de 10,4g. Quando maduros, têm a casca de coloração vermelho-intensa, com sulcos entre lóbulos bem marcantes. A polpa apresenta-se de coloração vermelha. Análises químicas dos frutos apresentaram teores de até 2.022,5 mg de ácido ascórbico, acidez titulável de 1,155 expressa em gramas de ácido cítrico por $100 \mathrm{~g}$ de polpa, com sólidos solúveis de $9,92^{\circ}$ Brix. Além destas características fisico-químicas, os frutos desta variedade apresentam um período de pós-colheita prolongado quando comparados com os de outras cultivares (DONADIO, 2000).

A composição química dos frutos depende da cultivar, das condições ambientais e também do estádio de maturação. No caso da acerola, a coloração é o principal critério de julgamento do amadurecimento dos frutos.

Três tipos principais de pigmentos ocorrem nos produtos vegetais: clorofila, carotenoides e antocianinas. A perda de clorofila e, consequentemente, da cor verde indica maturidade. $\mathrm{O}$ verde intenso no fruto jovem perde intensidade até tornar-se verde-claro, ou quando há completa perda do verde, aparecem os pigmentos amarelos, vermelhos ou púrpuros (CHITARRA; CHITARRA, 2005). Em acerolas, a coloração vermelha obtida com o avanço da maturação é decorrente da degradação da clorofila e do aumento na concentração de antocianinas (LIMA et al., 2003).

O objetivo deste trabalho foi avaliar a qualidade dos frutos da aceroleira cv. Olivier em dois estádios de maturação.

\section{MATERIAL E MÉTODOS}

As amostras de frutos foram coletadas em janeiro de 2010, em um pomar comercial de acerola da chácara Nossa Senhora Aparecida, localizada no município de Junqueirópolis-SP, latitude $21^{\circ} 18^{\prime} 00^{\prime \prime}$, longitude $51^{\circ} 15^{\prime} 00^{\prime \prime}$ e 420 metros de altitude. O clima do município, de acordo com a classificação de Köeppen, é subtropical úmido, Cwa, com inverno ameno e seco, e verão quente e chuvoso. A precipitação média é de $1.400 \mathrm{~mm}$ anuais e temperatura média anual de $24^{\circ} \mathrm{C}$, com máxima de $38^{\circ} \mathrm{C}$ e mínima de $14^{\circ} \mathrm{C}$ (CLIMA DOS MUNICÍPIOS PAULISTAS, 2008). O pomar possui 400 plantas da cultivar Olivier, distribuídas em uma área de 1 ha, com espaçamento de 6 metros entre linhas e 4 metros entre plantas. Trata-se de um pomar implantado no ano de 2003 , que apresenta produtividade crescente, sendo que a média na safra de 2007/2008 foi de $128,5 \mathrm{~kg} /$ planta.

O delineamento experimental utilizado foi o inteiramente casualizado, sendo que os estádios de maturação (frutos semimaduros e frutos maduros) constituíram os tratamentos, e as plantas analisadas (10) representaram as repetições. Os dados foram submetidos à análise de variância, pelo teste $\mathrm{F}$, e as médias, comparadas pelo teste de Tukey, a $5 \%$ de probabilidade. O programa estatístico utilizado foi o SIGMASTAT 3.5.

As análises físico-químicas foram realizadas no laboratório do Departamento de Produção Vegetal da Faculdade De Ciências Agrárias - UNESP/ Botucatu.

As amostras de frutos de cada repetição foram submetidas à análise de cor, determinada por meio de colorímetro marca Konica Minolta, modelo Chroma meter CR -400, espaço de cor L* a* b*, por reflectância. A calibração foi realizada com placa branca padrão, seguindo as instruções do fabricante. Os parâmetros de cor medidos com relação à placa-padrão foram: luminosidade $\left(\mathrm{L}^{*}\right)$, que varia da cor preta $(0)$ à branca $(100)$; $\mathrm{a}^{*}$, que varia da cor verde $(-60)$ à vermelha $(+60)$ e b*, que varia da cor azul $(-60)$ à amarela $(+60)$. Estes parâmetros foram usados em cálculos para a determinação dos índices de cor: Hue (ângulo hue), que é um indicativo de tonalidade e Chroma, que define a intensidade de cor. A polpa foi submetida às análises químicas de Teor de sólidos solúveis (I.A.L., 1985); pH (I.A.L., 1985); 
Acidez titulável (I.A.L, 1985); açucares redutores (SOMOGYI adaptado por NELSON, 1944) e Ácido ascórbico (MAPA, 2006), e calculado o índice de maturação 'Ratio' (sólidos solúveis/acidez total).

\section{RESULTADOS E DISCUSSÃO}

A coloração dos frutos utilizados nas análises químicas pode ser observada na tabela 1. Para o índice de luminosidade $L^{*}$, houve diferença estatística entre os estádios de maturação, sendo que os frutos com maturação intermediária apresentaram as maiores médias. Vendramini e Trugo (2000), estudando a composição química da acerola em três estádios de maturação, observaram que a luminosidade dos frutos aumentou quando passou de fruto imaturo para maturação intermediária; no entanto, com o avanço da maturação, a luminosidade diminuiu. Com relação ao índice $\mathrm{a}^{*}$, não houve diferença significativa entre os tratamentos. Já para o índice $b^{*}$, houve diferença, sendo que os frutos maduros apresentaram menor intensidade de cor amarela que os semimaduros. Pode-se observar que o índice $b^{*}$ apresentou valores menores que o índice $\mathrm{a}^{*}$, demonstrando menor intensidade de cor amarela que de cor vermelha, uma característica positiva da cultivar, já que hoje o mercado busca frutos com coloração avermelhada, mais atrativa ao consumidor.

Para os índices Chroma e Hue, os frutos semi-aduros também apresentaram os maiores valores. O maior valor de Chroma indica que estes frutos apresentaram cores mais vívidas que os frutos maduros. No entanto, os valores de Hue, indicativo de tonalidade, mostram que os frutos semimaduros, com valor de Hue de 39,81, apresentaram cor laranja, enquanto os frutos maduros, com valor de Hue de 30,08 , apresentaram cor próxima ao vermelho (Figura 1). Os dados de cor confirmam a diferença do estádio de maturação entre os tratamentos.

Na tabela 2, são apresentados os dados de qualidade das acerolas colhidas nos dois estádios de maturação. Pode-se observar um valor de $\mathrm{pH}$ maior nos frutos maduros que nos semimaduros, condição que se inverte para o índice acidez. $\mathrm{O}$ aumento do $\mathrm{pH}$ está diretamente relacionado com o decréscimo da acidez, ocorrida com o avanço da maturação dos frutos. Também nos frutos maduros foram encontradas as maiores médias para os índices de sólidos solúveis, 'ratio' e açúcares redutores. Para o índice de ácido ascórbico, os frutos maduros apresentaram médias bastante inferiores quando comparados com os frutos semimaduros (810,2 e 1738,5, respectivamente). Este resultado concorda com diversos autores (LIMA et al. 2005; NOGUEIRA et al. 2002; VENDRAMINI: TRUGO, 2000) De maneira geral, o teor de vitamina $\mathrm{C}$, expressa em ácido ascórbico, diminui com o avanço da maturação dos frutos e, em acerolas, esta diminuição é bem acentuada, visto que a maturação dos frutos ocorre de maneira rápida. $\mathrm{O}$ decréscimo da vitamina $\mathrm{C}$ durante o processo de maturação dos frutos ocorre devido à atuação da enzima ácido ascórbico oxidase (ascorbato oxidase), isolada em acerola por Asenjo et al. (1960), os quais verificaram que a atividade enzimática é maior nos frutos maduros. Apesar da diferença entra os tratamentos, os teores de ácido ascórbico obtidos neste trabalho são satisfatórios e estão de acordo com os encontrados em diversos trabalhos com acerola (ARAUJO et al., 2007; CARPENTIERE-PÍPOLO: BRUEL, 2002; NOGUEIRA et al., 2002).

TABELA 1 - Valores da determinação de cor $\left(L^{*}, a^{*}, b^{*}\right.$, Chroma e Hue) de acerolas cv. Olivier em dois estádios de maturação (Fruto maduro e semimaduro).

\begin{tabular}{cccccc}
\hline \multirow{2}{*}{$\begin{array}{c}\text { Estádio de } \\
\text { maturação }\end{array}$} & $\mathbf{L}^{*}$ & $\mathbf{a}^{*}$ & $\mathbf{b}^{*}$ & Chroma & Hue \\
\cline { 2 - 6 } & $40,12 \pm 2,53 \mathrm{~b}$ & $37,16 \pm 3,13 \mathrm{a}$ & $21,70 \pm 3,68 \mathrm{~b}$ & $43,15 \pm 4,14 \mathrm{~b}$ & $30,08 \pm 3,29 \mathrm{~b}$ \\
Maduro & $4,70 \pm 2,42 \mathrm{a}$ & $35,91 \pm 3,87 \mathrm{a}$ & $30,18 \pm 3,78 \mathrm{a}$ & $47,38 \pm 4,95 \mathrm{a}$ & $39,81 \pm 2,46 \mathrm{a}$ \\
Semimaduro & 44,70 aracterísticas de cor & $<0,172$ & $<0,001$ & $<0,001$ & $<0,001$ \\
\hline $\mathbf{P}$ & $<0,001$ & 0,17 &
\end{tabular}

Médias seguidas pela mesma letra na coluna não diferiram estatisticamente entre si, pelo teste de Tukey $(\mathrm{P}<0,05)$ 
TABELA 2 - Médias e desvio-padrão para as características químicas: pH, acidez titulável (AT), sólidos solúveis (SS), 'ratio'(SS/AT), açucares redutores (AR) e ácido ascórbico (AA) de frutos de acerola cv. Olivier, em dois estádios de maturação (fruto maduro e semi-maduro).

\begin{tabular}{cccc}
\hline \multicolumn{4}{c}{ Estádio de maturação } \\
\hline Maduro & Semimaduro & P \\
\hline PH & $3,68 \pm 0,05 \mathrm{a}$ & $3,56 \pm 0,05 \mathrm{~b}$ & $<0,001$ \\
AT & $3,15 \pm 0,15 \mathrm{~b}$ & $3,26 \pm 0,13 \mathrm{a}$ & 0,003 \\
SS & $7,58 \pm 0,35 \mathrm{a}$ & $7,42 \pm 0,25 \mathrm{~b}$ & 0,048 \\
'RATIO' & $2,41 \pm 0,13 \mathrm{a}$ & $2,27 \pm 0,08 \mathrm{~b}$ & $<0,001$ \\
AR & $5,73 \pm 0,56 \mathrm{a}$ & $4,24 \pm 0,48 \mathrm{~b}$ & $<0,001$ \\
AA & $810,2 \pm 53,3 \mathrm{~b}$ & $1738,5 \pm 189,6 \mathrm{a}$ & $<0,001$ \\
\hline
\end{tabular}

Médias seguidas pela mesma letra na linha não diferiram estatisticamente entre si, pelo teste de Tukey $(\mathrm{P}<0,05)$

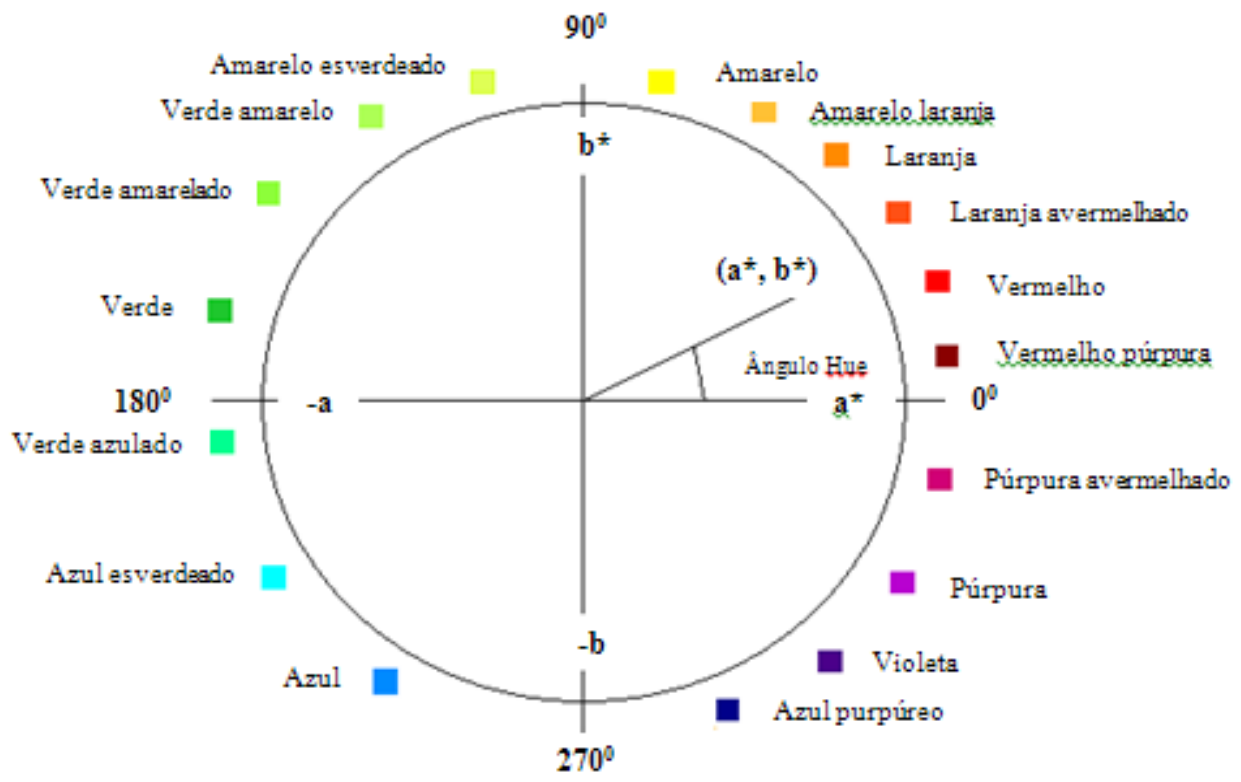

FIGURA 1 - Diagrama CIELAB com a sequência de nuances de cores e orientação do ângulo de nuances (Ângulo Hue). (Adaptado de CHITARRA : CHITARRA, 2005).

\section{CONCLUSÕES}

1. Os frutos maduros apresentaram teores de sólidos solúveis, açúcares redutores e $\mathrm{pH}$ maiores e teor de ácido ascórbico e acidez total menores que os frutos semimaduros.

2. Quando se buscam altos índices de Vitamina C, é recomendável que os frutos sejam colhidos num estádio de maturação menos avançado, ainda com coloração alaranjada.

3. Os frutos da aceroleira cv. Olivier possuem características adequadas tanto para o mercado in natura quanto para a indústria de suco, apresentando boa coloração e características químicas dentro dos padrões para esta fruta.

\section{REFERÊNCIAS}

AGRIANUAL: anuário da agricultura brasileira. São Paulo: FNP Consultoria e Comércio, 2010. 520 p.

ARAUJO, P. G. L. de; FIGUEIREDO, R. W. de; ALVES, R. E. ; MAIA, G. A. ; PAIVA, J. R. de. B-caroteno, ácido ascórbico e antocianinas totais em polpa de frutos de aceroleira conservada por congelamento durante 12 meses. Ciência e Tecnologia de Alimentos, Campinas, v. 27, n. 1, p. 104-107, 2007. 
ASENJO, C. F.; PENAlOZA, A.; MEDINA, P. Characterization of ascorbase present in the fruit of the Malphigiapunicifolia L. Federation of America Societies for Experimental Biology. Federation Proceedings, Bethesda, v.29, n.1, p.1, 1960.

CARPENTIERE-PÍPOLO, V.; BRUEL, D.C. Correlações fenotípicas, genotípicas e ambientais em aceroleira. Revista Brasileira de Fruticultura, Jaboticabal, v. 24, n. 1, p. 115-119, 2002.

CHITARRA, M. I. F.; CHITARRA, A. B. Pós-colheita de frutas e hortaliças: fisiologia e manuseio. 2 ed. Lavras: UFLA, 2005. 785 p.

CLIMA DOS MUNICÍPIOS PAULISTAS. Disponível em: <http://www.cpa.unicamp.br/outras-informacoes/clima_muni_297.html >. Acesso em: 02 nov. 2008.

IAL. Instituto Adolfo Lutz. Normas analíticas: métodos químicos e físicos para a análise de alimentos. 2.ed. São Paulo: 1985. v. 1, 371 p.

KONRAD, M. Efeito de sistemas de irrigação localizada sobre a produção e qualidade da acerola (Malpighiaspp) na região da Nova Alta Paulista. 2002. 119 p. Dissertação (Mestrado) - Faculdade de Engenharia, Universidade Estadual Paulista, Ilha Solteira, 2002.

LIMA, V. L. A. G. de; MÉLO, E. de A.; MACIEL, M. I. S.; LIMA, D. E. da S. Avaliação do teor de antocianinas em polpa de acerola congelada proveniente de frutos de 12 diferentes aceroleiras (Malpighiaemarginata. D.C.). Ciência e Tecnologia de Alimentos, Campinas, v. 23, n. 1, p. 101-103, 2003.

LIMA, V. L. A. G. de; MÉLO, E. de A. ; MACIEL, M. I. S.; PRAZERES, F. G.; MUSSER, R. S.; LIMA, D. E. da S. Total phenolicandcarotenoid contentes in acerola genotypesharvestedatthreeripeningstages. Food Chemistry, London, v.90, p.565-568, 2005.
MANICA, I.; ICUMA, I.M.; FIORAVANÇO, J.C.; PAIVA, J.R. de; PAIVA, M.C.; JUNQUEIRA, N.T.V. Acerola: tecnologia de produção, pós-colheita, congelamento, exportação, mercados. Porto Alegre: Cinco continentes, 2003. 397 p.

MAPA. Ministério da Agricultura, Pecuária e Abastecimento. Método de Tillmans modificado. Disponível em: <http://www.agricultura.gov.br>. Acesso em: 20 nov. 2006.

NELSON, N. A fotometria adaptationofsomogimethod for determinationof glicose. Journal Biological Chemistry, Baltimore, v. 31, n. 2, p. 159-161, 1944.

NOGUEIRA, R.J.M.C.; MORAES, J.A.P.V. de; BURITY, H.A.; SILVA JUNIOR, J.F. Efeito do estádio de maturação nas características físico-químicas da acerola. Pesquisa Agropecuária Brasileira, Brasília, v. 37, n. 4, p. 463-470, 2002.

RITZINGER, R.; RITZINGER, C.H.S.P. Acerola: aspectos gerais da cultura. Cruz das Almas: Embrapa Mandioca e Fruticultura Tropical, 2004. 2p. (Boletim Técnico)

TEIXEIRA, A.H. de C.; AZEVEDO, P.V. de. Potencial agroclimático do estado de Pernambuco para o cultivo da acerola. Revista Brasileira de Agrometeorologia, Santa Maria, v.2, p.105-113, 1994.

VENDRAMINI, A. L.; TRUGO, L. C. Chemicalcompositionof acerola fruit (Malpighiapunicifolia L.) atthreestagesofmaturity. Food Chemistry, London, v.71, p.195-198, 2000. 